\title{
Five-year prospective study on cardiovascular events, in patients with erectile dysfunction and hypotestosterone
}

\author{
Rosanna Iacona $^{1}$, Vito Bonomo ${ }^{1}$, Mariaconcetta Di Piazza ${ }^{1}$, Angela Sansone ${ }^{1}$, Manuela Usala ${ }^{2}$, \\ Salvatore Novo ${ }^{1}$, Carlo Pavone ${ }^{3}$ \\ ${ }^{1}$ Chair and Division of Cardiology, University Hospital "Paolo Giaccone", Palermo, Italy; \\ ${ }^{2}$ Orthopedic Institute Rizzoli - Department of Sicily Region (DRS), Bologna, Italy; \\ ${ }^{3}$ Chair and Division of Urology, University Hospital "Paolo Giaccone", Palermo, Italy.
}

\begin{abstract}
Summary Objective: Testosterone levels play a role in cardiac and vascular pathology. In the present study we investigated the prognostic significance of this hormone for cardiovascular outcome, in a 5-year follow-up. Materials and methods: Our cohort included 802 adult subjects, from 40 to 80 years. Patients were excluded if they had a past history of peripheral or coronary artery disease, and revascularization. A blood sample was drawn to valuate testosterone level, and we considered normal testosterone levels $300 \mathrm{ng} / \mathrm{dl}$. FMD (flow mediated dilatation) of the brachial artery was assessed by measuring the increase of the brachial artery diameter during reactive hyperemia after transient forearm ischemia. B-mode longitudinal images of the brachial artery were obtained at the level of the antecubital fossa.

The FMD was defined as the percentage change in the brachial artery diameter $60 \mathrm{~s}$ after releasing the ischemic cuff. Erectile dysfunction (ERD) was assessed by the International Index of Erectile Function-5 (IIEF-5) score questionnaire. We considered composite end points including the following major adverse cardiovascular events (MACEs)

Results: Subjects with lower serum testosterone levels ( $n=$ 332) had higher prevalence of traditional cardiovascular risk factors, such as hypertension $(p=0.009)$, diabetes $(p=0.03)$, dyslipidemia $(p<0.0001)$, obesity $(p=0.002)$, and endothelial function score $(p<0.0001)$. AMI, death after AMI, major stroke and all clinical events were more frequent $(p<0.001)$ in patients with testosterone levels $<300 \mathrm{ng} / \mathrm{dl}$. Further, by multiple logistic regression analysis we found that only dyslipidemia $(p=0,001)$, obesity $(p=0,007)$, testosterone $<300$ $n g / d l(p<0,0001)$ and ED ( $p<0,0001)$ were independent predictors of future events.

Conclusions: A therapeutic intervention on testosterone may not only have a positive effect on the cardiovascular system but also an important role in preventing new cardiovascular events.
\end{abstract}

KEY WORDS: Erectile dysfunction; Cardiovascular events; Prevention.

Submitted 22 August 2017; Accepted 23 October 2017

\section{INTRODUCTION}

Endothelial dysfunction (ED) represents the earliest event in the development of atherosclerotic plaque, and it actually occurs when a structural lesion is still not evident. Clinical and experimental evidence suggests that testosterone levels play a role in cardiac and vascular patholo- gy (1). In particular, in the present study we investigated the prognostic significance of this hormone for cardiovascular outcome, in a 5-year follow-up.

We aimed to assess whether baseline testosterone levels may be predictors of future cardiovascular events in a cohort of intermediate cardiovascular risk patients, according to Framingham risk score, and screened for the presence of ED in our Centre of Early Diagnosis of Preclinical and Multifocal Atherosclerosis and for Cardiovascular Prevention. The follow-up period was 5 years. We therefore aimed to test the hypothesis that measuring levels of this hormone, can provide information not only on the progression of atherosclerosis disease and therefore about ED, helping to define the prognosis, but can also be considered predictive of the risk of developing cardiovascular events, independently from common risk factors.

\section{Materials AND Methods}

Our cohort included 802 adult subjects, males from 40 to 80 years (mean age of the whole population was 57.52 \pm 13.85 years old). They were intermediate cardiovascular risk patients, according to Framingham risk score, all referred between 2009 and 2014 to our Department of Cardiology. Patients were excluded if they had a past history of peripheral artery disease, coronary revascularization, angina pectoris, myocardial infarction, carotid surgery or cerebrovascular event. The adopted procedures were in agreement with the Helsinki Declaration of 1975 as revised in 1983 and were approved by the local Ethic Council. All patients gave their informed consent to participate to the study, and at admission answered a questionnaire on personal and medical items, including age, past medical history and use of medications. The main cardiovascular risk factors were considered.

A blood sample was drawn in the morning, after 12-14 $\mathrm{h}$ overnight fast, to valuate testosterone level, and we considered normal testosterone levels N300 ng/dl (2, 3). Flow mediated dilatation (FMD) of the brachial artery was assessed (4) by measuring the increase of the brachial artery diameter during reactive hyperemia after transient forearmischemia. B-mode longitudinal images of the brachial artery were obtained at the level of the antecubital fossa. 
After marking the optimal position of the transducer, baseline images of the brachial artery were digitally stored. Arterial flow to the forearm was interrupted by insufflation of the forearm cuff for $5 \mathrm{~min}$ by $200 \mathrm{mmHg}$ or $50 \mathrm{mmHg}$ above systolic blood pressure, whichever was highest.

The FMD was defined as the percentage change in the brachial artery diameter $60 \mathrm{~s}$ after releasing the ischemic cuff.

Erectile dysfunction (ERD) was assessed by the International Index of Erectile Function-5 (IIEF-5) score questionnaire, which was preferentially filled in by the patient himself or, if required, by a trained interviewer. The possible score for the IIEF-5 ranges from 5 to 25, and ERD can be classified into five categories based on the score: severe (5-7), moderate (8-11), mild-to-moderate (12-16), mild (17-21), and no ERD (22-25) (5).

The median follow-up time for major CV events was 5.1 years. Except for 13 participants (excluded from the population) who moved abroad, there was no loss to follow-up in the study.

We considered composite end points including the following major adverse cardiovascular events (MACEs): acute myocardial infarction (AMI), death for AMI, minor stroke, major stroke, and death for stroke.

\section{RESULTS}

Baseline clinical characteristics, laboratory data and ultrasonography findings in relation to plasma values of testosterone are summarized in Table 1. Subjects with lower serum testosterone levels ( $\mathrm{n}=$ 332) had higher prevalence of traditional cardiovascular risk factors, such as hypertension ( $p=0.009)$, diabetes ( $p=0.03)$, dyslipidemia ( $p$ $<0.0001)$, obesity $(p=0.002)$, and endothelial function score ( $\mathrm{p}<$ 0.0001). There were no significant differences in age and in the presence of smoking or familiarity.

At five years follow-up we had 47 (5.8\%) AMI and 27 (3.45\%) minor or major stroke. $3.98 \%$ was the cardiovascular mortality. AMI,

Table 1. to IIEF-5 $(n=802)$. death after AMI, major stroke and all clinical events were more frequent $(\mathrm{p}<0.001)$ in patients with testosterone levels $<300 \mathrm{ng} / \mathrm{dl}$ at baseline (Figure 1).

Table 2 shows the baseline clinical characteristics, laboratory data and ultrasonographic findings in relation to the occurrence of all clinical events $(n=90)$, as registered in the 5-year follow-up. Further, by multiple logistic regression analysis we found that, among all evaluated baseline clinical and laboratory variables, only dyslipidemia ( $p=0,001)$, obesity $(p=0,007)$, testosterone < $300 \mathrm{ng} / \mathrm{dl}(\mathrm{p}<0,0001)$ and ED $(\mathrm{p}<0,0001)$ were independent predictors of future events.

\section{Discussion}

Several studies had stressed the relation between lower serum testosterone levels and erectile dysfunction, which is also a result of ED.

ED is therefore the common etiological factor for erectile dysfunction and cardiovascular events. In our study we wanted to define not only the known relation between ED and a worse erectile function in patients with lower levels of testosterone, but we also evaluate if this hormone could have an influence on cardiovascular outcome, beyond traditional risk factors and preclinical atherosclerosis. There is actually increasing evidence that

Relations between total serum testosterone, FMD and ED according

\begin{tabular}{|c|c|c|c|c|c|}
\hline & No ED & $\begin{array}{l}\text { Mild } \\
\text { ED }\end{array}$ & $\begin{array}{c}\text { Mild to } \\
\text { moderate ED }\end{array}$ & $\begin{array}{l}\text { Moderate } \\
\text { ED }\end{array}$ & $\begin{array}{l}\text { Severe } \\
\text { ED }\end{array}$ \\
\hline \multicolumn{6}{|c|}{ Total serum testosterone } \\
\hline Model 1 & 1.02 & 0.98 & 0.90 & 0.85 & 0.78 \\
\hline OR (Cl 95\%) & (0.89 to 1.16 ) & (0.64 to 1.10$)$ & (0.78 to 1.02$)^{* *}$ & (0.72 to 0.97$)^{* * *}$ & (0.62 to 0.86$)^{* * *}$ \\
\hline Model 2 & 1.03 & 0.95 & 0.88 & 0.83 & 0.80 \\
\hline OR (Cl 95\%) & (0.91 to 1.2 ) & (0.63 to 1.12 ) & $(0.80 \text { to } 1.06)^{* *}$ & $(0.77 \text { to } 0.95)^{* * *}$ & $(0.58 \text { to } 0.86)^{* * *}$ \\
\hline \multicolumn{6}{|l|}{ FMD (\%) } \\
\hline Model 1 & 1.04 & 0.94 & 0.81 & 0.76 & 0.68 \\
\hline OR (Cl 95\%) & (0.96 to 1.18$)$ & $(0.82 \text { to } 1.07)^{*}$ & $(0.69 \text { to } 0.94)^{* * *}$ & $(0.63 \text { to } 0.83)^{* * *}$ & (0.59 to 0.79$)^{* * *}$ \\
\hline Model 2 & 1.06 & 0.96 & 0.84 & 0.73 & 0.70 \\
\hline OR (Cl 95\%) & (1.02 to 1.22$)$ & (0.87 to 0.98$) *$ & $(0.70 \text { to } 0.93)^{* * *}$ & (0.66 to 0.79$)^{* * *}$ & (0.64 to 0.73$)^{* * *}$ \\
\hline \multicolumn{6}{|c|}{$\begin{array}{l}\text { FMD: flow-mediated vasodilation; OR: odd ratio; Cl: confidence inverval; ED: erectile dysfunction; } \\
\text { IIEF-5: international index of erectile function-5; FMD: artery flow-mediated vasodilation. } \\
\text { Model 1: adjusted for age. Model 2: adjusted for age and cardiovasclular risk factors. } \\
\star P<0.05 . \star \star P<0.01 .{ }_{\star \star \star \star P}<0.001 \text {. }\end{array}$} \\
\hline
\end{tabular}

Table 2.

Predictors of cardiovascular events at follow up.

\begin{tabular}{|c|c|c|c|c|c|c|}
\hline & \multicolumn{3}{|c|}{ Univariate logistic regression } & \multicolumn{3}{|c|}{ Multiple regression } \\
\hline & Odd ration & $95 \% \mathrm{Cl}$ & $\mathbf{P}$ & Odd ration & $95 \% \mathrm{Cl}$ & $\mathbf{P}$ \\
\hline Diabetes & 2.37 & 1.7797 to 3.1623 & $<0.0001$ & & & \\
\hline Hypertension & 4.14 & 3.0584 to 5.6145 & $<0.0001$ & & & \\
\hline Current smoking & 1.34 & 1.0218 to 1.7823 & 0.03 & & & \\
\hline Familiarity & 1.38 & 1.0485 to 1.8294 & 0.02 & & & \\
\hline Dyslipidemia & 4.14 & 3.0584 to 5.6145 & $<0.0001$ & 2.21 & 1.3944 to 3.5325 & 0.001 \\
\hline Testosterone $\leq 300 \mathrm{ng} / \mathrm{dl}$ & 10.10 & 7.2609 to 14.0694 & $<0.0001$ & 4.90 & 2.9971 to 8.0251 & $<0.0001$ \\
\hline Erectile function (score) & 0.75 & 0.7275 to 0.7890 & $<0.0001$ & 0.86 & 0.8165 to 0.9094 & $<0.0001$ \\
\hline Endothelial function (\%) & 0.81 & 0.7891 to 0.8434 & $<0.0001$ & 0.87 & 0.8321 to 0.9201 & $<0.0001$ \\
\hline
\end{tabular}


Figure 1.

Major adverse cardiovascular events (MACEs) at mean follow-up of 5.1 years according to testosterone levels.

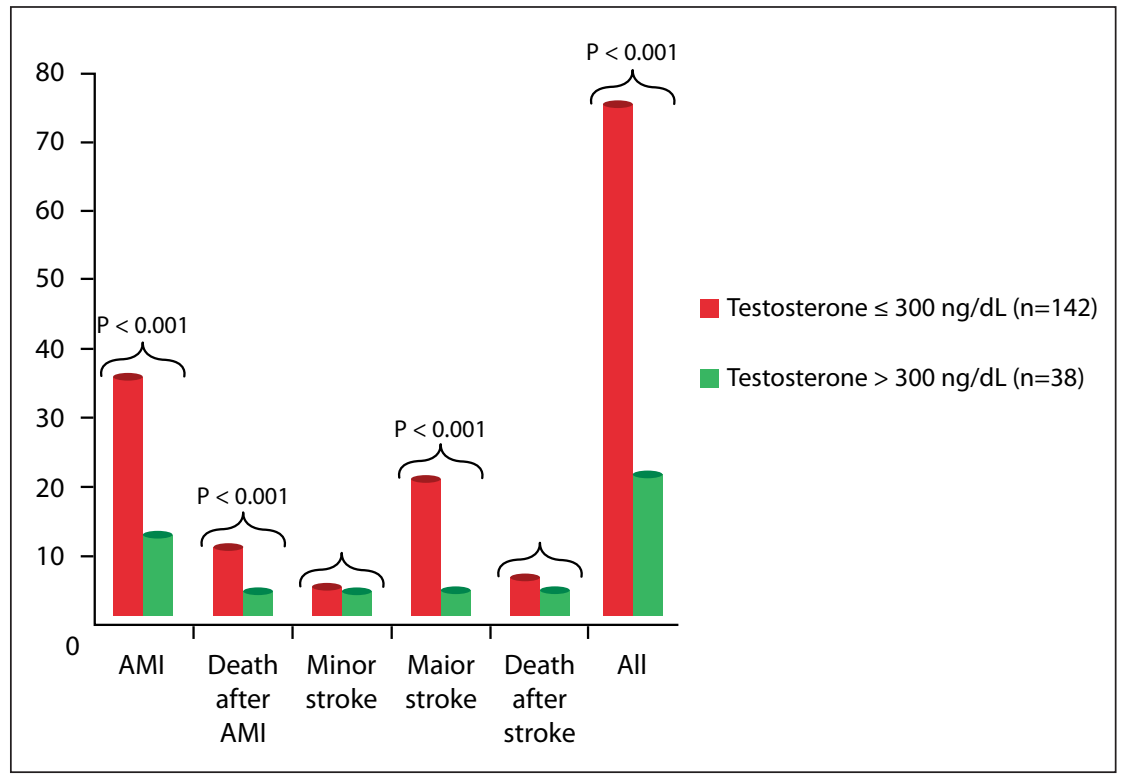

simplified International Index of Erectile Function (IIEF-5) as a diagnostic tool to study the prevalence of erectile dysfunction. Int J Impot Res. 2002; 14:245-250.

6. Sharma R, Oni OA, Gupta K, et al. Normalization of testosterone level is associated with reduced incidence of myocardial infarction and mortality in men. Eur Heart J. 2015; 36:2706-2715.

7- Vlachopoulos C, Jackson G, Stefanadis $C$, Montorsi P. Erectile dysfunction in the cardiovascular patient. Eur Heart J. 2013; 34:2034-2046.

8. Gencer B, Mach F. Testosterone: a hormone preventing cardiovascular disease or a therapy increasing cardiovascular events? Eur Heart J 2015.

9. Pavone C, Curto F, Anello G, et al. Prospective, randomized, crossover comparison of sublingual apomorphine $(3 \mathrm{mg})$ with oral sildenafil $(50 \mathrm{mg})$ for male erectile dysfunction. J Urol. 2008; 179(5 Suppl):S92-4. doi: 10.1016/j. juro.2008.03.144. PMID: 18405767. low testosterone levels are associated with increased cardiovascular mortality in men (6-9).

On the other hand, there was an association of low testosterone levels with decreased FMD independent of major cardiovascular confounders, so low levels of this hormone may be associated with impaired ED as measured by FMD.

\section{Conclusions}

Given the demonstrated relationship to events at the follow up, a possible therapeutic intervention on testosterone (with attention to the potentially increased risk of prostate cancer that remains a matter of debate), may not only have a positive effect on the cardiovascular system but also an important role in preventing newcardiovascular events, through a more accurate stratification of patients.

\section{REFERENCES}

1. Novo S, Iacona R, Bonomo V, et al. Erectile dysfunction is associated with low total serum testosterone levels and impaired flowmediated vasodilation in intermediate risk men according to the Framingham risk score, Atherosclerosis 2015; 238:415-419.

2. Haring R, Völzke H, Steveling A, et al. Low serum testosterone levels are associated with increased risk of mortality in a populationbased cohort of men aged 20-79, Eur Heart J. 2010; 31:1494-1501.

3. Laaksonen DE, Niskanen L, Punnonen K, et al. Testosterone and sex hormone-binding globulin predict the metabolic syndrome and diabetes in middle-aged men, Diabetes Care. 2004; 27:1036-1041.

4. Corretti MC, Anderson TJ, Benjamin EJ, et al. Guidelines for the ultrasound assessment of endothelial-dependent flow-mediated vasodilation of the brachial artery: a report of the International Brachial Artery Reactivity Task Force, J Am Coll Cardiol. 2002; 39:257-265.

5. Rhoden EL, Telöken C, Sogari PR, Vargas Souto CA. The use of the

\section{Correspondence}

Rosanna Iacona, MD (Corresponding Author) rosanna.iacona@virgilio.it

Vito Bonomo, MD vito_bonomo@alice.it

Mariaconcetta Di Piazza, MD mconcettadipiazza@libero.it

Angela Sansone, MD

angela.sansone2609@gmail.com

Salvatore Novo, MD

sav.novo@libero.it

Chair and Division of Cardiology, University Hospital "Paolo Giaccone" Via del Vespro 129, 90127 Palermo, Italy

Manuela Usala, MD

manuela.usala@ior.it

Orthopedic Institute Rizzoli - Department of Sicily Region (DRS)

Via Giulio Cesare Pupilli 1, 40136 Bologna, Italy

Carlo Pavone, MD

carlo.pavone@unipa.it

Chair and Division of Urology, University Hospital "Paolo Giaccone",

Via del Vespro 129, 90127 Palermo, Italy 